\title{
Bioprosthetic valve fracture to facilitate valve-in-valve transcatheter aortic valve repair
}

\author{
Keith B. Allen ${ }^{1}$, Adnan K. Chhatriwalla ${ }^{1}$, John T. Saxon ${ }^{1}$, Janarthanan Sathananthan ${ }^{2}$, Danny Dvir ${ }^{3,4}$, \\ John G. Webb ${ }^{2}$ \\ ${ }^{1}$ Saint Luke's Mid America Heart Institute and University of Missouri-Kansas City School of Medicine, Kansas City, MO, USA; ${ }^{2}$ Centre for Heart \\ Valve Innovation, St. Paul's Hospital and University of British Columbia, Vancouver, Canada; ${ }^{3}$ Shaare Zedek Medical Centre, Hebrew University, \\ Jerusalem, Israel; ${ }^{4}$ University of Washington, Seattle, WA, USA \\ Correspondence to: Keith B. Allen, MD. 4320 Wornall Road, Medical Plaza II, Suite 50, Kansas City, MO 64111, USA. Email: kallen2340@aol.com.
}

Submitted Jun 25, 2020. Accepted for publication Oct 07, 2020.

doi: $10.21037 /$ acs-2020-av-20

View this article at: http://dx.doi.org/10.21037/acs-2020-av-20

\section{Clinical vignette}

An 88-year-old female with a history of surgical aortic valve replacement twelve years earlier presented with New York Heart Association functional class IV heart failure in conjunction with a failing 21-mm Magna (Edwards Lifesciences, Irvine, CA) surgical valve. Echocardiography demonstrated an ejection fraction of $55 \%$ with a mean aortic valve gradient of $51 \mathrm{mmHg}$. Cardiac catheterization demonstrated no indication for coronary revascularization. She was evaluated by the heart team for reoperation versus valve-in-valve transcatheter aortic valve replacement (VIV TAVR).

The Society of Thoracic Surgeons predicated risk of mortality (STS PROM) with reoperation was $14 \%$. Computed tomography demonstrated adequate femoral access for TAVR. Risk of coronary obstruction during VIV TAVR was felt to be low with valve to coronary distance (VTC) of $>9 \mathrm{~mm}$ and a sinotubular junction diameter of $26 \mathrm{~mm}$. The heart team recommended VIV TAVR using a $23-\mathrm{mm}$ Sapien 3 (Edwards Lifesciences, Irvine, CA) transcatheter heart valve (THV). In addition, to minimize the risk of a high residual gradient following VIV TAVR in this small surgical valve and to optimize expansion of the THV, bioprosthetic valve fracture (BVF) using a $23 \mathrm{~mm}$ TRUE DILATION (Bard, Murry Hill, NJ) or Atlas Gold (Bard, Murry Hill, NJ) non-compliant balloon with a highpressure inflation was also recommended $(1,2)$.

\section{Surgical technique}

\section{Preparation}

VIV TAVR and BVF can be performed using either conscious sedation and transthoracic echocardiography (TTE) or general anesthesia and transesophageal echocardiography (TEE). Initially the pacing runs were longer if using a balloon expandable valve and general anesthesia/TEE was utilized. Currently pacing runs are not prolonged and conscious sedation/TTE is suitable. Bilateral 6-French femoral arterial sheaths are placed utilizing a micro puncture technique and a right ventricular pacing wire is positioned via transfemoral venous access. A pigtail catheter is positioned in the non-coronary sinus for imaging. Minimal contrast dye is required during VIV TAVR and the deployment angle is obtained by taking the parallax out of the surgical valve.

\section{Exposition}

Both self-expanding and balloon expandable THVs are suitable for VIV TAVR and BVF. While it was initially thought that the primary role of BVF would be to reduce high residual gradients following VIV TAVR and prevent patient-prosthesis mismatch, BVF may play an important additional role. By optimizing THV expansion and leaflet function, BVF may positively impact durability and may be beneficial regardless of the residual gradient during VIV 
TAVR (3). In selecting the size of the THV we anticipate a 3-4 $\mathrm{mm}$ increase in the diameter of the surgical valve after BVF (1). In the case above, the $21 \mathrm{~mm}$ Magna has a true internal diameter of $19 \mathrm{~mm}$ thus we would recommend using either a $23 \mathrm{~mm}$ Sapien 3 or $23 \mathrm{~mm}$ Evolut (Medtronic, Minneapolis, MN). The size of the non-compliant balloon used to perform BVF is important and should be at least 3 $\mathrm{mm}$ larger than the true internal diameter of the surgical valve in order to take full advantage of the potential expansion of surgical valve following fracture.

During initial bench testing, we used balloons that were $1 \mathrm{~mm}$ larger than the labeled valve size; however, in clinical practice, our goal is select a THV size and balloon size that will result in optimal expansion of the THV (1). In the case of Sapien 3 we try to 'right' size the non-compliant balloon in order to fully expand the Sapien 3. In this case we are using a $23 \mathrm{~mm}$ Sapien 3 and would plan to fracture with a $23 \mathrm{~mm}$ non-compliant balloon. As discussed in the video, when performing BVF following VIV TAVR with Evolut you should not use a non-compliant balloon more than $2 \mathrm{~mm}$ larger than the constrained waist of the THV, and the shoulder of the balloon should be kept at or below this area in order to avoid injury to the Evolut's leaflets (1). The timing of BVF remains controversial and the pros and cons of BVF before or after VIV TAVR are discussed in the video; however, we recommend performing BVF after VIV TAVR (2).

\section{Operation}

The 6-French sheath is exchanged over a stiff wire to the THV delivery sheath, the surgical prosthesis is crossed, baseline invasive hemodynamics are obtained and finally a coiled stiff wire is placed into the left ventricle. The appropriately orientated and crimped $23-\mathrm{mm}$ Sapien S3 is brought to the field and advanced to the thoracic aorta. The delivery balloon is then mounted and the THV is advanced and positioned for delivery. In order to try to minimize the residual gradient following VIV TAVR the implant depth, particularly for intra annular valves, should be very shallow (0-2 $\mathrm{mm})$, realizing that the Sapien 3 will foreshorten further during BVF.

In the case of balloon-expandable valves, rapid ventricular pacing is used to successfully deploy the valve. Following successful VIV TAVR, TEE demonstrated no paravalvular leak; however, invasive hemodynamics revealed a high residual gradient of $24 \mathrm{mmHg}$. BVF was performed uneventfully with a $23 \mathrm{~mm}$ Atlas Gold non-compliant balloon during rapid ventricular pacing with fracture occurring at 18 atmospheres. Final mean residual gradient was reduced to $9 \mathrm{mmHg}$.

\section{Completion}

We routinely extubate these patients at the conclusion of the case and recover them in our post-acute care unit for several hours before transferring to the floor. Patients typically are discharged home in 1-2 days.

\section{Comments}

\section{Clinical results and advantages}

VIV TAVR is an approved treatment option for patients with failed surgical bioprosthetic valves who are at prohibitive or high surgical risk; however, there remain concerns with VIV TAVR, particularly in patients suitable for reoperation. Elevated residual transvalvular gradients $(>20 \mathrm{mmHg}$ ) may occur following VIV TAVR, particularly in the presence of a small surgical bioprosthesis, which have been associated with worse clinical outcomes and reduced 1-year survival (4). In addition, VIV TAVR may result in the THV being constrained by the surgical valve resulting in suboptimal leaflet motion, which may impact durability (3).

BVF is a technique that utilizes a non-compliant balloon and high-pressure inflation to fracture the surgical valve and allow optimal expansion of the THV (1). In a large multicenter series, BVF was safely performed in conjunction with both balloon and self-expanding THV's and resulted in significantly lower final transvalvular residual gradients and increased valve effective orifice area (2). In addition, 1-year follow up demonstrates sustained low gradients, no signal for THV injury and improved survival compared to historical controls (5). While the timing of BVF is still controversial, performing BVF after VIV TAVR results in the lowest final mean transvalvular gradient, mitigates the risk of catastrophic aortic insufficiency from ballooning the degenerated surgical prosthesis and allows the case to be performed in a controlled, predictable fashion.

\section{Caveats}

While BVF can be performed safely with reduction in high residual gradients following VIV TAVR, its long-term impact on clinical outcomes and THV durability, either positive or negative, requires further study. In addition, 
whether all surgical valves, regardless of size and residual gradients, should be fractured to optimize transcatheter valve expansion is currently being debated. Finally, the expansion of BVF into the VIV mitral space where even small reductions in gradients may yield high benefits is only now being studied.

\section{Acknowledgments}

Funding: None.

\section{Footnote}

Conflicts of Interest: KBA-Edwards Lifesciences: Research Support, Proctor, Speakers Bureau; Medtronic: Research Support, Speakers Bureau; Abbott: Research Support, Consulting; Boston Scientific: Consulting. AKC-Abbott Vascular: Speakers Bureau; Boston Scientific: Research support, consulting; Edwards Lifesciences: Proctor, Speakers Bureau; Medtronic Inc: Proctor, Speakers Bureau. JS-Edwards Lifesciences and Medtronic: Consulting. DD-Consulting for Edwards Lifesciences, Medtronic, Abbott. JGW-Consulting for Edwards Lifesciences, Abbott, Boston Scientific. JTS has no conflicts of interest to declare.

Open Access Statement: This is an Open Access article distributed in accordance with the Creative Commons
Attribution-NonCommercial-NoDerivs 4.0 International License (CC BY-NC-ND 4.0), which permits the noncommercial replication and distribution of the article with the strict proviso that no changes or edits are made and the original work is properly cited (including links to both the formal publication through the relevant DOI and the license). See: https://creativecommons.org/licenses/by-nc-nd/4.0/.

\section{References}

1. Allen KB, Chhatriwalla AK, Cohen DJ, et al. Bioprosthetic Valve Fracture to Facilitate Transcatheter Valve-in-Valve Implantation. Ann Thorac Surg 2017;104:1501-8.

2. Allen KB, Chhatriwalla A, Saxon J, et al. Bioprosthetic Valve Fracture to Facilitate VIV TAVR: Insights from a Multicenter Study. J Thorac Cardiovasc Surg 2019;158:1317-28.e1.

3. Sathananthan J, Sellers S, Barlow AM, et al. Valve-in-Valve Transcatheter Aortic Valve Replacement and Bioprosthetic Valve Fracture Comparing Different Transcatheter Heart Valve Designs: An Ex Vivo Bench Study. JACC Cardiovasc Interv 2019;12:65-75.

4. Dvir D, Webb JG, Bleiziffer S, et al. Transcatheter aortic valve implantation in failed bioprosthetic surgical valves. JAMA 2014;312:162-70.

5. Chhatriwalla A, Allen KB, Saxon J, et al. One year outcomes following bioprosthetic valve fracture to facilitate valve-in-valve transcatheter aortic valve replacement. J Am Coll Cardiol 2020;75:1106.
Cite this article as: Allen KB, Chhatriwalla AK, Saxon JT, Sathananthan J, Dvir D, Webb JG. Bioprosthetic valve fracture to facilitate valve-in-valve transcatheter aortic valve repair. Ann Cardiothorac Surg 2020;9(6):528-530. doi: 10.21037/acs-2020av-20 